ISSN 0103-5150

Fisioter. Mov., Curitiba, v. 25, n. 3, p. 607-615, jul./set. 2012

Licenciado sob uma Licença Creative Commons

\title{
Especificidade e sensibilidade da radiografia digital da coluna lombar como ferramenta de auxílio ao diagnóstico da osteoporose
}

\author{
Lumbar spine digital radiography sensitivity and \\ specificity as a tool for the diagnosis of osteoporosis
}

\author{
Lelia Paes Jeronymo ${ }^{[a]}$, Munir Antonio Gariba ${ }^{[b]}$ \\ [a] Fisioterapeuta, mestre em Tecnologia em Saúde pela Pontifícia Universidade Católica do Paraná (PUCPR), docente da \\ Faculdade Guairacá, Guarapuava, PR - Brasil, e-mail: paeslelia@bol.com.br \\ [b] Físico, doutor em Engenharia Biomédica pelo Imperial College of Science, Technology and Medicine, Reino Unido; \\ docente do Programa de Pós-Graduação em Tecnologia em Saúde da Pontifícia Universidade Católica do Paraná \\ (PUCPR), Curitiba, PR - Brasil, e-mail: munir.gariba@pucpr.br
}

Resumo

Introdução: A osteoporose é considerada um distúrbio osteometabólico que tem como característica a diminuição da densidade mineral óssea e a deterioração da microarquitetura do tecido ósseo, o que ocasiona aumento da fragilidade esquelética e do risco de fraturas. A técnica adotada como padrão-ouro para quantificar massa óssea é a densitometria óssea (DXA). Porém, o alto custo e o consequente baixo acesso da população ao exame dificultam o diagnóstico, o que leva a maior número de pacientes com fraturas e suas consequências. Objetivo: 0 objetivo desse trabalho é estudar a sensibilidade e a especificidade da radiografia digital da coluna lombar como ferramenta de auxílio ao diagnóstico da osteoporose. Metodologia: 0 estudo foi realizado com 64 pacientes, sendo 4 do gênero masculino e 60 do feminino, avaliados por radiografia digital e por densitometria quanto à presença ou não de osteopenia ou osteoporose. As radiografias foram analisadas independentemente por três radiologistas experientes e no caso de divergência no laudo, foi considerada a classificação modal. Resultados: Conforme o coeficiente Kappa, o grau de concordância entre as técnicas foi baixo, sendo a sensibilidade e a especificidade da radiografia para o diagnóstico de osteopenia/osteoporose estimadas em 85\% e 16,7\%, respectivamente. Conclusão: A radiografia digital mostrou ser uma técnica sensível para verificar se a condição da vértebra no exame apresenta-se normal 
ou alterada, porém se mostrou pouco específica para distinguir entre osteopenia e osteoporose. Apesar da especificidade da densitometria, a radiografia mostrou ser o melhor método quando há fraturas vertebrais.

Palavras-chave: Osteoporose. Densitometria. Radiografia. Doenças ósseas metabólicas.

\section{Abstract}

Introduction: Osteoporosis is considered an osteometabolic disorder that is characterized by the reduction of bone mineral density and deterioration of bone microarchitecture resulting in an increase of bone fragility and fracture risk. The technique considered as gold standard to measure bone mineral density is Dual energy X-ray Absorptiometry (DXA). Nevertheless, high cost and consequently the limited access to the modality by the population make the diagnosis difficult, leading to a higher number of patients with fracture and its consequences. Objective: The general objective of this paper is to study the viability of digital radiography of lumbar spine as an auxiliary diagnostic tool for osteoporosis. Methodology: This study was carried out with 64 patients (4 male and 60 female) assessed by digital radiography and densitometry concerning the presence of osteoporosis or osteopenia. The radiographies were analyzed independently by three experienced radiologists, and modal classification was considered in case of report divergence. Results: According to the Kappa coefficient, the agreement level between these two techniques is low. Radiography sensitivity and specificity for osteopenia/osteoporosis diagnosis was estimated as $85 \%$ and $16.7 \%$, respectively, considering densitometry as the gold standard. Conclusion: Digital radiography showed to be a sensitive technique to verify if vertebra condition is normal or altered, however, it is little specific in the distinction between osteopenia and osteoporosis. Densitometry was more specific in the diagnosis of osteopenia and osteoporosis, but in cases with complication such as vertebra fracture, the radiography showed to be the best method.

Keywords: Osteoporosis. Densitometry. Radiography. Bone Diseases Metabolic.

\section{Introdução}

A osteoporose é considerada um distúrbio osteometabólico que tem como característica a diminuição de densidade mineral óssea e deterioração da microarquitetura do tecido ósseo, o que ocasiona aumento da fragilidade esquelética e risco de fraturas (1). A maioria dos indivíduos acometidos são mulheres na pós-menopausa, mas homens e mulheres jovens também podem apresentar a doença (2). As fraturas são as principais manifestações clínicas da osteoporose, sendo que as vértebras, o fêmur e os ossos do antebraço são os locais mais frequentemente afetados (3).

Pode-se classificar a osteoporose em primária (idiopática) ou secundária. A forma primária é classificada em tipo I e tipo II. A do tipo I, ou pós-menopausa, está associada às fraturas das vértebras e do radio distal, resultado de rápida perda óssea, que afeta principalmente mulheres que acabaram de entrar na menopausa. A do tipo II, também conhecida como senil, surge com o envelhecimento do indivíduo, como resultado da deficiência crônica de cálcio, aumento da atividade do paratormônio e diminuição da formação óssea. A osteoporose secundária relaciona-se a processos inflamatórios, como a artrite reumatoide, alterações endócrinas, mieloma múltiplo, uso de drogas como heparina, álcool, vitamina A e corticoides (4), sendo relacionada também à anorexia nervosa, doença hepática crônica, síndrome do intestino irritável, doença celíaca, hipogonadotrofismo, doença renal e deficiência de vitamina D (5).

Atualmente, a osteoporose é considerada, nos países desenvolvidos, como um dos problemas mais sérios e comuns na população idosa, principalmente em mulheres. Os custos sociais da doença são altos em grande parte graças aos custos de fratura de fêmur (6-8). Nos Estados Unidos, o custo direto de fraturas osteoporóticas é superior a US $\$ 17$ bilhões por ano (9). No Brasil, de acordo com dados do Ministério da Saúde, são gastos com fraturas de quadril por osteoporose a cifra média de 28 milhões de reais por ano (10).

Autores sugerem que os profissionais da saúde devem ajudar a identificar os riscos de pacientes com osteoporose sofrerem quedas e fraturas, identificando nesses pacientes fatores de risco importantes para osteoporose, como: história médica, exame físico e exames laboratoriais $(5,11)$.

Outros ressaltam que a detecção precoce da osteoporose é muito importante. A osteoporose é 
uma doença que pode ser tratada e prevenida (12, 13). A OMS recomenda que as pessoas realizem atividade física, mantenham o peso ideal, tenham uma dieta rica em cálcio e vitamina $\mathrm{D}$, evitem cigarro e excesso de álcool como medida de prevenção para reduzir o risco de osteoporose (14).

A técnica considerada padrão-ouro para a medida de massa óssea é a densitometria, constituindo-se num importante meio não invasivo para a avaliação de pacientes com osteoporose, possibilitando a realização de seu diagnóstico e seguimento. Mede-se o conteúdo mineral ósseo ou a densidade mineral areal, ou seja, a quantidade mineral dividida pela área óssea estudada (15), conforme ilustra a Figura 1.

A realização do exame de densidade óssea é indicada para mulheres com 65 anos ou mais, homens com 70 anos ou mais, mulheres acima de 40 anos na transição menopausal (1-2) e homens acima de 50 anos de idade com fatores de risco, adultos com história de fraturas por fragilidade, condição clínica ou uso de medicamentos associados à baixa massa óssea ou perda óssea, indivíduos para os quais são consideradas intervenções farmacológicas para osteoporose, pessoas em tratamento para osteoporose, para monitorar a eficácia do tratamento, indivíduos que não estão realizando tratamento, porém nos quais a identificação de perda de massa óssea possa determinar a indicação do tratamento e mulheres interrompendo terapia hormonal (TH) (16).

A Organização Mundial da Saúde (OMS) estabeleceu o diagnóstico da osteoporose com base no escore $\mathrm{T}$ da densitometria óssea, retirado de uma população de mulheres caucasoides na faixa etária entre 20 e 45 anos, conforme mostra a Tabela 1.

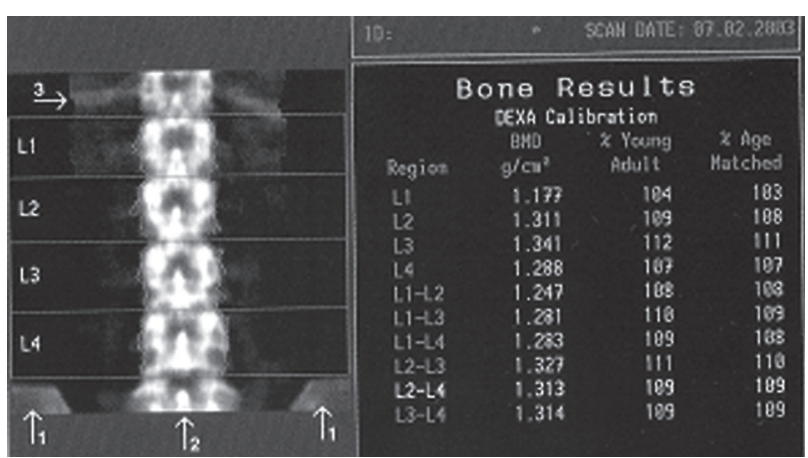

Figura 1 - Imagem de exame de densidade óssea quantificada por densitometria

Fonte: Dados da pesquisa.
0 rastreio de osteoporose por medição da DMO não é economicamente eficiente para mulheres na pós-menopausa. Sugere-se verificar a probabilidade de osteoporose a partir de testes mais baratos, verificando fatores de risco para osteoporose, tais como: dor nas costas, exame físico e resultado de radiografias (17).

A radiografia é a primeira modalidade empregada para se verificar a ocorrência de osteoporose, desencadeando novas investigações diagnósticas. Num estudo que analisa várias radiografias panorâmicas, verificou-se que elas podem ser importante ferramenta no diagnóstico de osteoporose em mulheres pós-menopausa (18). A avaliação radiológica não invasiva desempenha um importante papel no diagnóstico, tratamento e estudo da osteoporose, podendo identificar facilmente fraturas. Além da avaliação qualitativa, índices quantitativos têm sido empregados para determinação da densidade mineral óssea (19).

Em estudo correlacionando a radiografia simples do quadril com a densitometria óssea, observou-se que, embora as radiografias não sejam um teste para osteoporose, elas podem ser uma ferramenta útil para identificar pacientes em risco de fraturas (20). Porém, alguns autores analisam a radiografia tradicional como pouco sensível, capaz de detectar perda óssea somente quando esta atingiu níveis de 30-50\% (8).

Em muitos serviços de radiologia, a radiografia convencional está sendo substituída pela radiografia digital. Entretanto, estudos mostram que a taxa de detecção de osteoporose não apresenta diferença significativa na precisão diagnóstica entre a radiografia digital (Figura 2) a convencional (13).

Tabela 1 - Definição de osteoporose pela OMS, baseada em valores da densitometria óssea

\begin{tabular}{|c|c|}
\hline Definição & Valor da DMO \\
\hline Normal & $\begin{array}{l}\text { até -1DP da média do } \\
\text { adulto jovem }\end{array}$ \\
\hline Osteopenia & entre -1 e $-2,5 \mathrm{DP}$ \\
\hline Osteoporose & abaixo de $-2,5 \mathrm{DP}$ \\
\hline Osteoporose estabelecida & $\begin{array}{l}\text { abaixo de }-2,5 D P \text {, na presença } \\
\text { de uma ou mais fraturas por } \\
\text { fragilidade }\end{array}$ \\
\hline
\end{tabular}

Fonte: Anijar (15). 


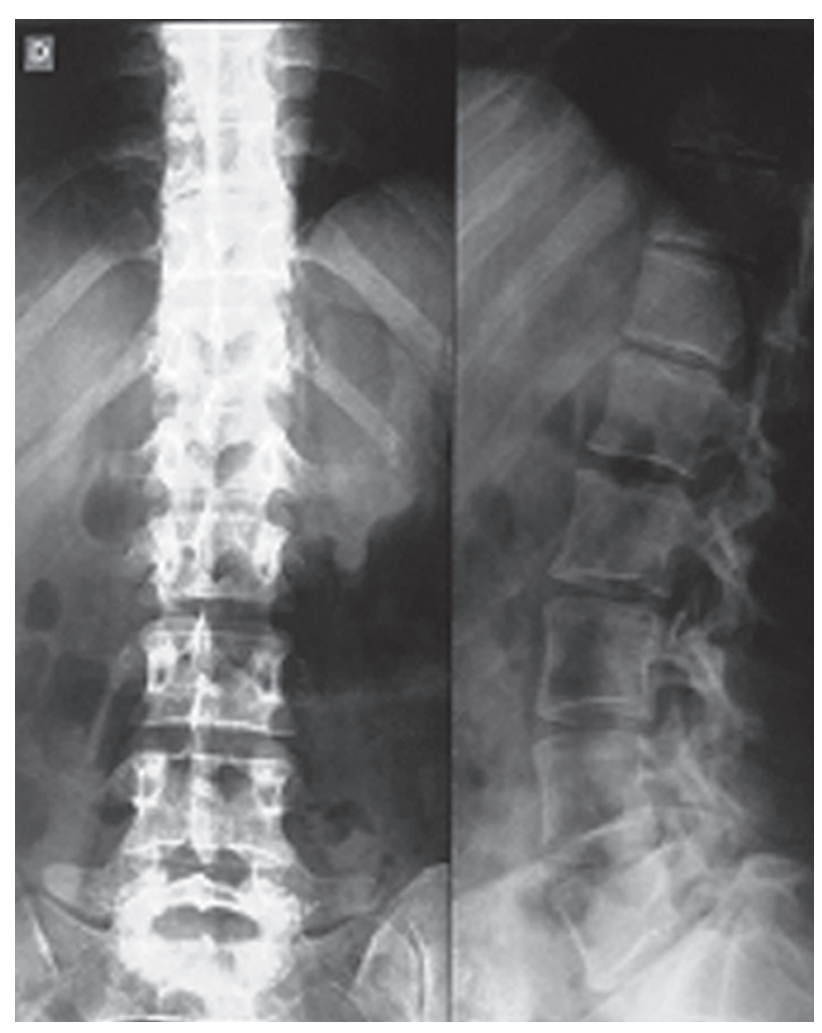

Figura 2 - Radiografia digital da coluna

Fonte: Dados da pesquisa.

\section{Metodologia}

Trata-se de um estudo de corte transversal, com exames de pacientes encaminhados para exame de densitometria pela técnica DXA, na Clínica da Imagem na cidade de Ponta Grossa, Paraná.

O estudo teve a aprovação do Comitê de Ética em Pesquisa da Pontifícia Universidade Católica do Paraná (CEP). Os voluntários assinaram o Termo de Consentimento Livre e Esclarecido (TCL). Foram incluídos 64 exames de pacientes, sendo 60 do gênero feminino e 4 do gênero masculino, avaliados por radiografia e por densitometria quanto à presença de osteopenia, presença de osteoporose ou normal. Os exames utilizaram os equipamentos disponíveis na Clínica da Imagem, a saber: densitometria no aparelho HOLOGIC QDR 4500C, realizada no procedimento de rotina da clínica, incluindo calibração diária, medições da coluna lombar de L1 a L4 e colo do fêmur; radiografia digital da coluna lombar em aparelho de raios-X da marca Philips ${ }^{\circledR} 500 \mathrm{ma}$, sendo as imagens adquiridas nas incidências anteroposterior (AP) e perfil.

As radiografias foram analisadas de forma independente por três médicos e, no caso de divergência no laudo, foi considerada a classificação modal (a mais frequente). 0 objetivo da análise foi avaliar a concordância entre os resultados das duas técnicas e avaliar a qualidade da radiografia no diagnóstico da osteopenia/osteoporose.

Para avaliar o grau de concordância entre a radiografia e a densitometria, foi estimado o coeficiente de Kappa e construído intervalo de 95\% de confiança para esse parâmetro. A qualidade da radiografia no diagnóstico de osteopenia/osteoporose foi avaliada estimando-se valores de sensibilidade e especificidade com intervalos de $95 \%$ de confiança. Os dados foram analisados com o programa computacional Statistica v. 8.0.

\section{Resultados}

Na análise da concordância radiografia/densitometria, a concordância entre as duas técnicas ocorreu em $30(46,9 \%)$ dos 64 casos.

Em 12 (18,7\%) casos, a radiografia subestimou o resultado em relação à densitometria (seis casos normais na radiografia e osteopenia na densitometria; seis casos de osteopenia na radiografia e osteoporose na densitometria).

Em $22(34,4 \%)$ casos, a radiografia superestimou o resultado em relação à densitometria (15 casos de osteopenia na radiografia e normal na densitometria; 5 casos de osteoporose na radiografia e normal na densitometria; 2 casos de osteoporose na radiografia e osteopenia na densitometria) conforme Tabela 2.

0 coeficiente de Kappa estimado para o grau de concordância entre a radiografia e a densitometria foi de 0,173 , com intervalo de $95 \%$ de confiança dado por 0 a 0,35 .

Na análise da concordância radiografia/densitometria (normal ou alterado), a concordância entre as duas técnicas ocorreu em 38 (59,4\%) dos 64 casos.

Em seis $(9,4 \%)$ casos, a radiografia subestimou o resultado em relação à densitometria Em 20 (31,2\%) casos, a radiografia superestimou o resultado em relação à densitometria, de acordo com a Tabela 3.0 coeficiente de Kappa estimado para o grau de concordância entre a radiografia e a densitometria é igual a 0,02 , com intervalo de $95 \%$ de confiança dado por 0 a 0,31 .

A sensibilidade da radiografia para o diagnóstico de osteopenia/osteoporose, considerando-se a densitometria como padrão-ouro, foi estimada em 85\%, 
com intervalo de $95 \%$ de confiança dado por $73,9 \%$ a $96,1 \%$.

A especificidade da radiografia para o diagnóstico de osteopenia/osteoporose, considerando-se a densitometria com padrão-ouro, foi estimada em $16,7 \%$, com intervalo de $95 \%$ de confiança dado por $1,8 \%$ a $31,6 \%$ (Tabela 4).

Com base na amostra do estudo, estima-se que $62,5 \%$ da população estudada têm densitometria alterada. Considerando-se esse resultado, o valor preditivo positivo para a radiografia foi estimado em $63 \%$, com intervalo de $95 \%$ de confiança dado por $50,1 \%$ a $75,8 \%$. 0 valor preditivo negativo para a radiografia foi estimado em $40 \%$, com intervalo de $95 \%$ de confiança dado por 9,6\% a 70,4\%.

No Quadro 1 são apresentados os resultados dos oito casos de maior discordância dentre os 64 estudados, conforme resultado da avaliação de cada médico e da densitometria. Na avaliação dos três médicos

Tabela 2 - Análise da concordância radiografia/densitometria

\begin{tabular}{lcccc}
\hline \multirow{2}{*}{ RX } & \multicolumn{3}{c}{ Densitometria } & Total \\
\cline { 2 - 4 } & Normal & Osteopenia & Osteoporose & \\
\hline Normal & 4 & 6 & 0 & 10 \\
& $6,25 \%$ & $9,38 \%$ & $0,00 \%$ & \\
Osteopenia & 15 & 18 & 6 & 39 \\
& $23,44 \%$ & $28,13 \%$ & $9,38 \%$ & \\
Osteoporose & 5 & 2 & 8 & 15 \\
& $7,81 \%$ & $3,13 \%$ & $12,50 \%$ & \\
Total & 24 & 26 & 14 & 64 \\
\hline
\end{tabular}

Fonte: Dados da pesquisa.

Tabela 3 - Análise da concordância radiografia/densitometria (normal ou alterado)

\begin{tabular}{lccc}
\hline \multirow{2}{*}{ RX } & \multicolumn{2}{c}{ Densitometria } & \multirow{2}{*}{ Total } \\
\cline { 2 - 3 } & Normal & Alterado & \\
\hline Normal & 4 & 6 & 10 \\
& $6,25 \%$ & $9,38 \%$ & \\
Alterado & 20 & 34 & 54 \\
& $31,25 \%$ & $53,13 \%$ & \\
Total & 24 & 40 & 64 \\
\hline
\end{tabular}

Fonte: Dados da pesquisa. especialistas, as classificações utilizadas para o laudo das radiografias foram: normal, osteopenia, osteoporose e osteoporose/fratura. Essa última significou que o paciente apresentou osteoporose já com a presença de fratura.

Dos 64 casos analisados, em oito $(12,5 \%)$ casos pelo menos um médico classificou o paciente como tendo osteoporose/fratura. Desses, na densitometria, dois foram classificados como normais, quatro como tendo osteopenia e apenas dois como tendo osteoporose.

\section{Discussão}

A osteoporose é hoje um grande problema de saúde pública, considerada como uma das doenças mais sérias e comuns na população idosa, principalmente do sexo feminino, podendo evoluir por anos sem sintomas até a ocorrência de uma fratura (21). Apesar dessa evolução silenciosa, sua consequência clínica de longo prazo e a ocorrência de fraturas tem um impacto significativo sobre a qualidade de vida, morbidade e mortalidade.

Graças ao envelhecimento da população, o número de idosos vem aumentando, assim como tem aumentado o número de pacientes com osteoporose e suas consequências negativas, tanto para o indivíduo como para a sociedade (22).

A osteoporose é uma doença que pode ser prevenida e tratada. Porém, o conhecimento em relação à doença é muito baixo, principalmente em homens, pois são os que menos percebem os sintomas (23).

Alguns autores definem a osteoporose como uma doença silenciosa (22). Contudo, outros afirmam que

Tabela 4 - Avaliação da qualidade da radiografia osteopenia/osteoporose

\begin{tabular}{lccc}
\hline \multirow{2}{*}{ RX } & \multicolumn{2}{c}{ Densitometria } & Total \\
\cline { 2 - 3 } & Normal & Alterado & \\
\hline Normal & 4 & 6 & 10 \\
& $16,7 \%$ (espec.) & & \\
Alterado & 20 & 34 & 54 \\
& & $85,0 \%$ (sensib.) & \\
Total & 24 & 40 & 64 \\
\hline
\end{tabular}

Fonte: Dados da pesquisa. 
Quadro 1 - Resultados dos radiologistas - osteoporose/ fraturas (comparação com densitometria)

\begin{tabular}{llll}
\hline Médico 1 & Médico 2 & Médico 3 & Densitometria \\
\hline Osteop/fratu & Osteop/fratu & Osteop/fratu & Osteopenia \\
Osteop/fratu & Osteopenia & Osteopenia & Osteopenia \\
Osteop/fratu & Osteop/fratu & Osteop/fratu & Normal \\
Osteoporose & Osteoporose & Osteop/fratu & Osteoporose \\
Osteoporose & Osteop/fratu & Osteop/fratu & Osteoporose \\
Osteop/fratu & Osteopenia & Osteopenia & Osteopenia \\
Osteopenia & Osteop/fratu & Osteopenia & Osteopenia \\
Osteop/fratu & Osteop/fratu & Osteoporose & Normal \\
\hline
\end{tabular}

Fonte: Dados da pesquisa.

a osteoporose, por ser uma doença mediada por osteoclastos, apresenta a característica de ser dolorosa, podendo a dor ser mais branda ou passar despercebida (24).

É necessário que os profissionais de saúde levem à população mais esclarecimento sobre a doença, seus fatores de risco e os métodos para sua detecção precoce (25).

A demora em atender a população é uma desvantagem da densitometria, sendo necessárias alternativas de métodos de diagnóstico, como, por exemplo, considerar fatores de risco e resultados da radiografia $(17,14)$. A radiografia convencional é a primeira modalidade a suspeitar de osteoporose (18). Além disso, a radiografia de coluna em pacientes apresentando fatores de risco para osteoporose poderá beneficiá-los, pois é possível iniciar o tratamento medicamentoso e reduzir o risco de novas fraturas de coluna e quadril.

Uma mulher de 65 anos com uma fratura de coluna tem uma chance em quatro de apresentar outra fratura em cinco anos, e esse número cai para uma chance em oito, se iniciar o tratamento (23).

Pesquisadores têm usado radiografias de quadril unilateral para diagnóstico de pacientes em risco de fraturas por osteoporose (20), porém, outros estudos sugerem que o exame de radiografia de fêmur seja realizado bilateralmente para não comprometer o diagnóstico (26).

No presente estudo, dos 64 casos estudados, a concordância entre as duas técnicas ocorreu em 30 (46,9\%). Dos 34 casos em que ocorreu discordância,
$12(18,7 \%)$, a radiografia subestimou o resultado em relação à densitometria (6 casos normais na radiografia e osteopenia na densitometria; $6 \mathrm{ca}$ sos de osteopenia na radiografia e osteoporose na densitometria).

Esses resultados estão de acordo com autores que afirmam que a radiografia é menos sensível para detectar perda de massa óssea, só ocorrendo quando essa atingir 30-50\% de perda óssea (27, $28,8)$. Outros afirmam que a radiografia desempenha papel importante no diagnóstico, tratamento e estudo da osteoporose, porém $20 \%$ de conteúdo mineral ósseo têm que ser perdido antes de ser visível a osteopenia (19).

Há relatos de que as alterações na radiografia aparecem quando a osteoporose está avançada. As mais visíveis são afilamento da cortical dos ossos longos com irregularidade da face endosteal e maior visibilidade do córtex mais fino, graças ao aumento de contraste com o osso desmineralizado. 0 mesmo é observado na vértebra, onde o padrão trabecular fica mais acentuado, pela perda das trabéculas horizontais e permanência das estruturas trabeculares verticais (29).

Em 22 casos $(34,4 \%)$, a radiografia superestimou o resultado em relação à densitometria (15 casos de osteopenia na radiografia e normal na densitometria; 5 casos osteoporose na radiografia e normal na densitometria; 2 casos de osteoporose na radiografia e osteopenia na densitometria).

Nesses casos, os resultados estão de acordo com Costa-Paiva et al. (12), quando sugerem que o exame de radiografia deve ser solicitado juntamente com o de densitometria, pois as medições efetuadas pela densitometria são bidimensionais e, quando realizadas em imagens adquiridas em projeção anteroposterior, que é a mais usada nesse procedimento, têm a desvantagem de medir as densidades de todos os componentes minerais encontrados em seu percurso, incluindo algumas alterações como processos degenerativos (osteófitos), calcificações vasculares e extravertebrais. Essas alterações podem influenciar no resultado da densidade mineral óssea, se as medições ocorrerem em regiões com processos degenerativos, e esses fatores podem levar a medições de massa óssea superestimadas em regiões afetadas.

A concordância entre as duas técnicas, considerando-se as classificações normais e alteradas, ocorreu em 38 dos casos $(59,4 \%)$. Em seis casos 
$(9,4 \%)$, a radiografia subestimou o resultado em relação à densitometria, mostrando que a técnica é pouco sensível para alterações e que essas alterações precisam estar em um nível mais elevado para serem diagnosticadas na radiografia. Porém, a sensibilidade da radiografia é menor quando se avalia somente osteopenia, sendo maior a concordância quando osteopenia/osteoporose recebem a mesma classificação. Em 20 casos (31,2\%), a radiografia superestimou o resultado em relação à densitometria, mostrando que quando a avaliação é somente entre alterado ou normal, a radiografia é mais sensível do que quando há necessidade de distinção dos casos alterados entre osteopenia e osteoporose.

A avaliação da qualidade da radiografia digital no diagnóstico de osteopenia/osteoporose mostrou uma sensibilidade de $85 \%$, ou seja, a radiografia mostrou-se sensível para diagnosticar osteopenia/ osteoporose, porém a especificidade da radiografia digital para o diagnóstico de osteopenia/osteoporose, considerando a densitometria padrão-ouro, foi estimada em $16,7 \%$. Ou seja, a radiografia digital não se mostrou específica para o diagnóstico de osteopenia/osteoporose.

Dos 64 casos analisados, em oito $(12,5 \%)$ ao menos um médico classificou o paciente como tendo osteoporose/fratura. Desses, quando avaliados pela densitometria, dois foram classificados como normais, quatro como tendo osteopenia e dois como tendo osteoporose.

No presente estudo, em oito casos a densitometria provavelmente subestimou o resultado em relação às radiografias, ao classificar como normal e osteopenia exames que, na radiografia, acusaram osteoporose com fraturas. Portanto, a radiografia mostrou-se mais sensível e específica para o diagnóstico de fraturas, se comparada à densitometria, concordando com estudos que consideram a técnica insensível e imprecisa para detectar fraturas vertebrais (30). Vale dizer que as localizadas acima das vértebras torácicas, já que a imagem DXA tem menor resolução, podem não mostrar doenças ou outros problemas visíveis na radiografia convencional. Outras análises sobre a qualidade da densitometria para o diagnóstico de fraturas concluíram que a técnica apresenta qualidade de imagem inferior e baixa sensibilidade para diagnóstico de fraturas leves (31).

\section{Conclusão}

Neste estudo, verificou-se que, conforme o índice de Kappa, ocorreu baixo grau de concordância entre a densitometria e a radiografia digital para o diagnóstico específico de osteopenia e osteoporose. Entretanto, de acordo com a prevalência de $62 \%$, pode-se concluir que ocorreu alto grau de concordância entre a densitometria e a radiografia digital para a determinação da existência de alguma dessas patologias, pois a radiografia digital mostrou ser uma técnica sensível para a distinção entre um exame normal e um alterado. Contudo, ela é pouco específica para permitir a distinção entre osteopenia e osteoporose.

A densitometria mostrou-se uma técnica mais específica para o diagnóstico de osteopenia e osteoporose. Porém, nos casos em que ocorrem complicações, como fraturas vertebrais, a radiografia mostrou ser um método diagnóstico sensível.

Sendo a radiografia uma técnica mais barata e mais acessível, sugere-se que, para pacientes que clinicamente apresentem maior chance de fratura, em virtude da idade ou por associação de fatores de risco, o exame radiológico seja utilizado para auxiliar no diagnóstico de osteoporose em larga escala anteriormente à possível indicação do exame de densitometria óssea.

\section{Referências}

1. Pinto-Neto AM, Soares A, Urbanetz AA, Araújo e Souza AC, Ferrari AEM, Amaral B, et al. Consenso brasileiro de osteoporose 2002. Rev Bras Reumatol. 2002;42(6):343-54.

2. Cubas ER, Boeving A, Marcatto C, Santos CMC, Borba VCZ, Kulak CAM. Principais causas de diminuição da massa óssea em mulheres na pré-menopausa encaminhadas ao ambulatório de doenças ósteo-metabólicas de um hospital terciário de Curitiba. Arq Bras Endocrinol Metab. 2006;50(5):914-19. doi:10.1590/ S0004-27302006000500013.

3. Damilakis J, Maris TG, Karantanas AH. An update on the assessment of osteoporosis using radiologic techniques. Eur Radiol. 2007;17(6):1591-602.doi:10.1007/ s00330-006-0511-z. 
4. Gali JC. Osteoporose. Acta Ortop Bras. 2001;9(2):53-62. doi:10.1590/S1413-78522001000200007.

5. Walker J. Osteoporosis, pathogenesis, diagnosis and management. Nurs Stand. 2008;22(17):48-56.

6. Araújo DV, Oliveira JHA, Bracco OL. Custo da fratura osteoporótica de fêmur no sistema suplementar de saúde brasileiro. Arq Bras Endocrinol Metabol. 2005;49(6):897-901. doi:10.1590/S000427302005000600007.

7. Cury AF, Zacchello KP. Osteoporose: prevalência e fatores de risco em mulheres de clínica privada maiores de 49 anos de idade. Acta Ortop Bras. 2007;15(3):146-50.

8. Silva LK. Avaliação tecnológica em saúde: densiometria óssea e terapêuticas alternativas na osteoporose pós-menopausa. Cad Saúde Pública. 2003;19(4):987-1003. doi:10.1590/S0102-311X2003000400022.

9. Lewiecki EM, Borges JLC. Densidade óssea na prática clínica. Arq Bras Endocrinol Metabol. 2006;50(4):586-95.

10. Secretaria da Saúde. A osteoporose. 2011. [citado em 12 out. 2011]. Disponível em: http://www.saude.df.gov. $\mathrm{br} /$.

11. Mcclung B. Clinical evaluation of osteoporosis and fracture risk in postmenopausal women. Clin Rev Bone Miner Metab. 2006;4(1):15-23.

12. Costa-Paiva L, Horovitz AP, Santos AO, Fonsechi-Carvasan GA, Pinto-Neto AM. Prevalência de osteoporose em mulheres na pós-menopausa e associação com fatores clínicos e reprodutivos. Rev Bras Ginecol Obstet. 2003;25(7):507-12. doi:10.1590/ S0100-72032003000700007.

13. Wagner S, Stäbler A, Sittek H, Bonel H, Laeverenz G, Reiser MF, et al. Diagnosis of osteoporosis: visual assessment on conventional versus digital radiographs. Osteoporos Int. 2005;16(12):1815-22. doi.10.1007/ s00198-005-1937-x.

14. Woolf AD. The global perspective of osteoporosis. Clin Rheumatol. 2006;25(5):613-8. doi:10.1007/ s10067-006-0265-7.

15. Anijar JR. Densitometria óssea na prática médica. São Paulo: Sarvier; 2003.

16. Brandão CMA, Camargos BM, Zerbini CA, Plapler PG, Mendonça LMC, Albergaria BH, et al. Posições Oficiais 2008 da Sociedade Brasileira de Densitometria Clínica (SBDens). Arq Bras Endocrinol Metab. 2009;53(1):10712. doi:10.1590/S0004-27302009000100016.
17. Ofluoglu D, Gunduz OH, Bekirolu N, Kul-Panza E, Akyuz G. A method for determining the grade of osteoporosis based on risk factors in postmenopausal women. Clin Rheumatol. 2005;24(6):606-11. doi:10.1007/ s10067-005-1110-0.

18. Leite AF, Figueiredo PTS, Guia CM, Melo NS, Paula AP. Radiografia panorâmica: instrumento auxiliar no diagnóstico de osteoporose. Rev Bras Reumatol. 2008;48(4):226-33. doi:10.1590/S048250042008000400006 .

19. Kowalchuk RM, Dalinka MK. The radiologic assessment of osteoporosis. Orthop J. 1998;11:67-72.

20. Sah AP, Thornhill TS, Leboff MS, Glowacki J. Correlation of plain radiographic indices of the hip with quantitative bone mineral density. Osteoporos Int. 2007;18(8):1119-26. doi:10.1007/ s00198-007-0348-6.

21. Guarniero R, Oliveira LG. Osteoporose atualização no diagnóstico e princípios básicos para o tratamento. Rev Bras Ortop. 2004;39(9):477-85.

22. Netelenbos JC, Lems WF, Geusens PP, Verhaar HJ, Boermans AJM, Boomsma MM, et al. Spine radiographs to improve the identification of women at high risk for fratures. Osteoporos Int. 2009;20(8):1347-52. doi:10.1007/s00198-008-0801-1.

23. Doheny MO, Sedlak CA, Estok PJ, Zeller R. Osteoporosis knowledge, health beliefs, and DXA T- Scores in men and women 50 years of age and older. Orthop Nurs. 2007;26(4):243-50. doi:10.1097/01. NOR.0000284654.68215.de.

24. Souza MPG. Diagnóstico e tratamento da osteoporose. Rev Bras Ortop. 2010;45(3):220-9.

25. Kaptoge S, Armbrecht G, Felsenberg D, Lunt M, ONeil TW, Silman AJ, et al. When should the doctor order a spine X-Ray? Identifying vertebral fractures for osteoporoses care: results from the European prospective osteoporosis study (EPOS). J Bone Miner Res. 2004;19(12):1982-93. doi:10.1359/jbmr.040901.

26. Alele JD, Kamen DL, Hermayer KL, Fernandes J, Soule J, Ebeling M, et al. The prevalence of significant left-right hip bone mineral density differences among black and white women. Osteoporos Int. 2009;20(12):2079-85. doi:10.1007/s00198-009-0915-0.

27. Wyngaarden JB, Smith LH. Cecil Tratado de medicina interna. 16a ed. Rio de Janeiro: Interamericana; 1984. p. 1353-55. 
28. Meirelles ES. Diagnóstico por imagem na osteoporose. Arq Bras Endocrinol Metab. 1999;43(6):423-7. doi:10.1590/S0004-27301999000600006.

29. Edeiken J. Roentgen diagnosis of diseases of bone. Baltimore: Williams \& Wilkins; 1981.

30. El Maghraoui A, Roux C. DXA scanning in clinical practice. Q J Méd. 2008;101(8):605-17. doi:10.1093/qjmed/ hen 022 .
31. Fuerst T, Wu C, Genant HK, Von Ingersleben G, Chen Y, Johnston C, et al. Evaluation of vertebral fracture assessment by dual x-ray absorptiometry in a multicenter setting. Osteoporos Int. 2009;20(7):1199-205. doi:10.1007/ s00198-008-0806-9.
Recebido: 30/11/2011

Received: $11 / 30 / 2011$

Aprovado: 20/07/2012

Approved: 07/20/2012 